\title{
Fluid-Structure Interaction of a Spring Mounted Symmetrical Rigid Wing for Drag Reduction of Cars at Higher Wind Velocities
}

Jason Knight, Simon Fels, George Haritos \& Thomas Carolus

\begin{abstract}
This paper details an aeroelastic concept for an adaptive and passive wing, which is primarily aimed for use within automotive sector to reduce drag and fuel emissions. The work will also be of interest in the motorsport sector to improve performance and also some applications within aerospace and renewable energy sectors. The wind tunnel testing of a spring mounted symmetrical NACA 0012 wing in freestream is studied over $0^{\circ}$ to $40^{\circ}$ angles of incidence. General operation of the concept is verified at low angles in the prestall region with that of a theoretical estimation using finite and infinite wings. Three distinct regions are identified, pre-stall, near-stall and post-stall. The transient limitations associated in the near-stall region with variations in spring loading and flow velocities are discovered. It is identified as a periodic self-sustained oscillation with non-dimensional reduced frequencies in a range of 0.14 to 0.22 . Furthermore, performance in the post-stall region along with pre-stall is reported and methods for the adjustment of the elastic element for a desired response are introduced. Evaluation is conducted with regard to an automotive application such as a rear wing on a high downforce race car. Typically a $25 \%$ increase in wind velocity in the pre-stall region results in a $3^{\circ}-5^{\circ}$ change in angle of incidence corresponding to a $25-40 \%$ reduction of drag coefficient depending on spring stiffness. Reductions of $20^{\circ}$ in angle of incidence with similar $25 \%$ increase in wind velocity are typically found in the post-stall region. Even larger reductions are found when transitioning through the stall region. This work provides a valuable insight for a novel concept, but we only recommend its use in the pre-stall region to achieve steady results. Use at higher angles is only recommended if transient effects are not important. Limitations to this proof of concept work are highlighted and future development work is suggested to achieve further increases in performance.
\end{abstract}


Keywords: Fluid-structure interaction, NACA0012, drag reduction, aerodynamic optimisation, stability.

\section{Nomenclature}

$$
\begin{aligned}
& A R=\text { aspect ratio } \\
& c=\text { chord length } \\
& C_{l} \quad=\quad \text { lift coefficient } \\
& f=\text { oscillation frequency } \\
& g=\text { gravitational acceleration } \\
& K=\text { reduced frequency }(f c \pi) / v \\
& L \quad=\quad \text { lift force } \\
& m_{w} \quad=\quad \text { mass of the wing } \\
& m_{s} \quad=\quad \text { mass of the sample weight for calibration of the spring } \\
& \operatorname{Re} \quad=\quad \text { Reynolds number }\left(\rho_{\text {Air }} v c\right) / \mu \\
& S \quad=\quad \text { span length } \\
& v=\text { wind velocity } \\
& x_{A C}=\text { distance from aerodynamic centre to the leading edge } \\
& x_{C o G}=\text { distance from centre of gravity to the leading edge } \\
& x_{M A}=\text { distance from mounting axis to the leading edge } \\
& \alpha=\text { angle of incidence } \\
& \eta \quad=\quad \text { aerodynamic efficiency factor } \\
& \theta=\text { spring preload angle at no angle of incidence } \\
& \kappa=\text { torsional spring constant of the spiral spring } \\
& \rho_{\text {Air }}=\text { air density } \\
& \tau_{a}=\text { Aerodynamic moment about the mounting axis } \\
& \tau_{a, \alpha=0}=\text { torque setting }\left(\tau_{s}-\tau_{g} \text { where } \alpha=0\right) \\
& \tau_{g}=\text { moment caused by gravity about the mounting axis } \\
& \tau_{s}=\text { spring torque about the mounting axis }
\end{aligned}
$$




\section{Introduction}

Modern race cars use wings and diffusers to increase downforce. Diffusers typically increase downforce and reduce $\operatorname{drag}^{1}$ whereas wings typically increase both downforce and drag. ${ }^{2}$ High downforce is desired in corners on the one hand, while higher top speed on the straights is facilitated by lower drag on the other hand. Thus, the development of wings faces a conflict between low drag and high downforce particularly on high-speed circuits. Active aerodynamic systems can address this issue by adapting downforce and drag with moveable wings and flaps. ${ }^{3}$ Furthermore, theses active aerodynamic systems can be utilised to lower the drag and thus lower the fuel consumption, ${ }^{4}$ which is of particular interest on production cars.

One of the first cars that implemented the concept of a wing to generate downforce was the Chaparral 2E, which was introduced in 1966 for the Can-Am series. ${ }^{5}$ A giant conventional airplane wing was used upside down. Furthermore, the wing had a hydraulic system to adjust the angle of incidence to a low drag position. ${ }^{6}$ The low drag position was manually initiated by an extra pedal. When the pedal was released, the wing returned to its normal high downforce position.

Modern race and sports cars utilise actively adjustable aerodynamics to gain the best driving performance. Nowadays, electro-mechanical actuators or hydraulic systems are used to tilt the wing. In Formula One for example, the Drag Reduction System (DRS) was introduced in 2011 to facilitate overtaking manoeuvres. ${ }^{6}$ Strict rules to regulate the use of the DRS are specified by the Federation Internationale de l'Automobile. ${ }^{7}$ Active rear wing systems that include a low drag functionality are also implemented in current super sports cars. $^{8,9}$

Aeroelasticity is the study of the effect of aerodynamic forces acting on an elastic body. ${ }^{10}$ Most research published in this area is understandably targeted at aerospace engineering applications. Researchers have focused on flexible wing structures that incorporate the use of composite materials. ${ }^{11,12}$ Composite structures open up the design space for tailoring the stiffness in a defined direction. In this way, higher divergence and flutter speeds, improved gust response and drag reduction can be achieved. Stodieck et al. ${ }^{11}$ applied the latest 
advances in tow-steered composites, which allow for a free orientation of the fibres within each plie. This proved to be even more efficient than composites with traditional straight fibres. More recently, De Breuker et al. ${ }^{13}$ published a unified framework for aeroelastic tailoring of composite aircraft wings. Weisshaar and Duke ${ }^{14}$ utilised aeroelastic tailoring for drag reduction of control surfaces. An adaptive concept to improve the lift-to-drag ratio of an unmanned air vehicle (UAV) by changing the relative position of internal spar webs to adjust the torsional stiffness was investigated by Ajaj et al. ${ }^{15}$ Earlier research on flexible wing structures at lower Reynolds numbers is found in the field of micro air vehicles (MAV) ${ }^{16}$ Recently, aeroelastic tailoring has been applied to enhance the performance of wind turbine systems. ${ }^{17}$ In automotive engineering, Thuwis et al. ${ }^{18}$ conducted a study on a fixed flexible Formula One rear wing to reduce drag. This was achieved by a wash-out effect of the wing resulting from a bending-torsion coupling. The bending-torsion coupling was controlled by modification of the lamination parameters of the skin of the wing. The result of this study was a successful drag reduction at high speeds while preserving the low speed performance.

Ghommem et al. ${ }^{19}$ studied an aeroelastic setup similar to ours and computed the transient effects in the near-stall region. To the authors knowledge there is no comparable experimental aeroelastic system, which incorporates a rigid wing with an external elastic component, which allows for the adjustment of it. Although sharing the same aim as active aerodynamic systems, this work focuses on a different approach of aerodynamic adaptation.

The proposed concept is based on intentionally induced deformation of an elastic element. It is envisioned, that the adaptation is only triggered at high driving speeds, while the downforce is fully maintained during lower speed cornering. The angle of incidence of the wing shall be lowered at a defined movement speed, which is selectable in advance. Therefore, the concept is considered adjustable and passive, but not active. It promises to be simple, lightweight and cost-effective, while maintaining the adaptive drag functionality of active aerodynamic systems.

The symmetrical NACA 0012 wing profile is used in this work to allow for comparison with extensive published literature. Dynamic stall characteristics of the NACA 0012 airfoil 
were observed in experiments by McCrokskey et al. ${ }^{20}$ and McAllister et al. ${ }^{21}$ and in simulations by Akbari \& Price. ${ }^{22}$ The dynamic stall shows distinct characteristics at certain Reynolds numbers of current interest in this work. These include the formation of a laminar separation bubble (LSB) and reattachment. ${ }^{23}$ The LSB is expected only near the stall region and not at angles of incidence greater than $15^{\circ}$. Above this, a completely separated airflow is expected. ${ }^{24}$

D. Poirel et al. ${ }^{25}$ investigated the oscillations of an aeroelastic NACA 0012 airfoil and also suggests the presence of LSBs. While their experimental setup was capable of pitch and plunge motion, our focus is on their results on pitch motion only. A steady-state selfsustained oscillation was observed at Reynolds numbers in the range of $4.5 \times 10^{4}$ up to 1.3 $\times 10^{5}$. We follow a similar aeroelastic setup as Poirel et al., ${ }^{25}$ except we mount the wing in the horizontal plane, use a preloaded spring and include the effect of weight.

The main objective of the current work focuses on testing, validating and analysing the fundamental principal of the concept, not on the drag reduction itself. Throughout this work, we mount the wing on a pitching axis that is at $10 \%$ of the chord. This location is always upstream of the aerodynamic centre, so that a higher speed will tend to reduce the angle of incidence resulting in lower coefficients of lift and drag. Using a mounting axis downstream of the aerodynamic centre will have the opposite effect and increase both lift and drag coefficients. This would potentially be of interest in automotive engineering whereby high speed stability is of prime concern, rather than lift and drag reduction. It would also be of interest in the work of using shielding fences to protect the workforce whilst maintaining some ventilation. ${ }^{26}$ However, this is beyond the current scope of work, whereby we concentrate on reducing aerodynamic forces with higher wind velocities.

The present work is also motivated by the question of whether it is possible to trigger the rotation of the wing at a desired velocity. Furthermore, it shall be investigated if the system is vulnerable to oscillation about the rotational axis. Ultimately, it must be examined whether such a concept for an adaptive and passive wing is recommended for an automotive and motorsport application or not. It is also important to mention, production and race cars are subject to vibrations and accelerations in all three directions, but these are not considered in the current work along with the effect of varying the moment inertia. In 
addition, the flow around road vehicles is non-uniform, but we only consider uniform flow in this proof of concept work. Also beyond the scope of this paper is the effect of damping on the motion of the wing.

\section{Methodology}

A mechanical solution is proposed to achieve the goal of a rear wing that self-adjusts to the driving speed. The angle of incidence of the wing shall be lowered at high driving speeds, resulting in a reduced drag. The realisation of the proposed system is based on mounting the wing on a rod with a rotational degree of freedom in the pitch axis only. A spiral spring with adjustable preload, $\theta$ which will serve as the elastic element, is attached to the rod. The required force for the rotation of the wing is provided by the aerodynamic pitching moment due to the airflow, once a certain preload of the spring is overcome. Thus, the angle of incidence, $\alpha$ is dependent on the wind velocity, weight of wing and preload in the spring. A schematic of the system is shown in Figure 1. The experiments reported later include various spring preload settings at increasing airflow velocities, whereas the weight remains constant throughout this work.

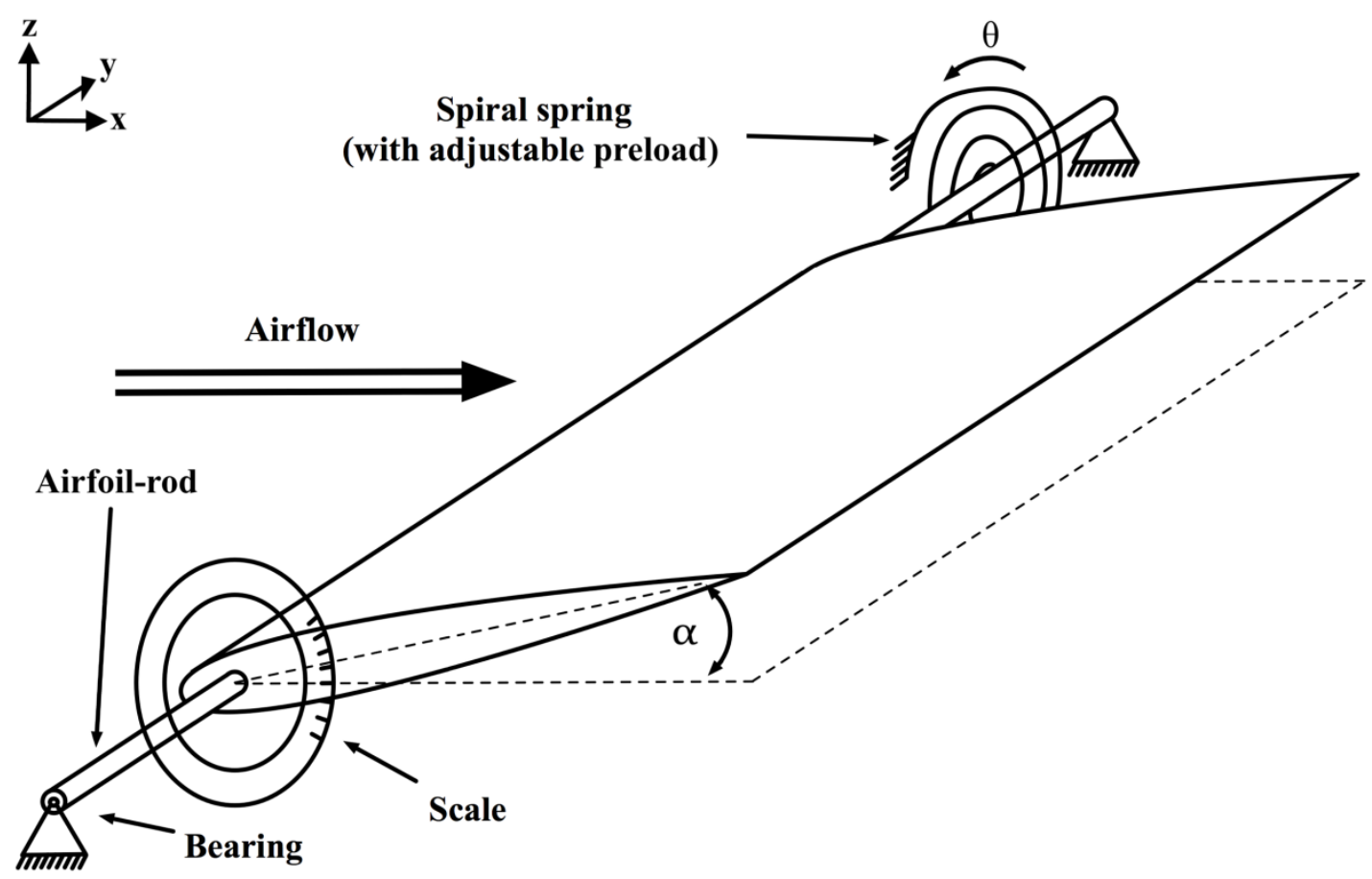

Figure 1) Schematic of the experimental setup. 


\subsection{Characteristics of the mechanical system}

The moment about the mounting axis resulting from the airflow $\tau_{a}$, the spring torque $\tau_{s}$ and the moment of the wing weight, $\tau_{g}$ represent the total moment of this system assuming negligible friction. In a static equilibrium, the total moment about the mounting axis will equal zero. Thence,

$$
\tau_{s}+\tau_{g}+\tau_{a}=0
$$

(Equation 1)

In this work, we take the rotational direction of these moments to be positive leading edge up. The moment due to gravitation, $\tau_{g}$ varies with the angle of incidence, $\alpha$ and is calculated according to,

$$
\tau_{g}(\alpha)=\cos (\alpha)\left(x_{C o G}-x_{M A}\right) m_{w} g
$$

(Equation 2)

where $m_{w}$ is the mass of the wing, $g$ is the acceleration due to gravity, $x_{C o G}$ is location of centre of gravity and $x_{M A}$ is the location of mounting axis, both measured form leading edge. Second, the spring torque $\tau_{s}$ is defined. The spring shall only be used in the linearelastic range. Therefore, Hooke's Law can be applied to calculate $\tau_{s}$ with $\kappa$ being the torsional spring constant, so that,

$$
\tau_{s}(\theta, \alpha)=-\kappa(\theta-\alpha)
$$

(Equation 3)

where $\theta$ is the spring preload angle and $\alpha$ is the angle of incidence. Tensioning the spring by a certain angle leads to a restoring force, which is acting counter clockwise. Thus, the right hand side of Equation 3 is defined as negative. The preload angle is defined by a counter clockwise rotation of the outer fixing point of the spiral spring on the one hand and a stationary airfoil at no angle of incidence on the other hand. The angle of incidence shall then be subtracted, as it is defined in the same rotational direction. 
The aerodynamic moment driving the rotation of the symmetrical wing is next examined. The lift force, drag and pitching moment about the aerodynamic centre are considered. In the special case of a symmetrical airfoil, there is no pitching moment about the aerodynamic centre, which is consistent with published experimental data that shows a pitching moment about the quarter-chord very close to zero. ${ }^{27}$ The aerodynamic centre is defined as the point about which the aerodynamic moment is not changing with angle of incidence. Aerodynamic moment arising from drag is commonly not considered in aeroelastic theory ${ }^{28,29}$ and for the sake of simplicity we also ignore it here. This assumption holds in low angles of incidence and relatively high lift to drag ratios. In this case of a thin symmetrical airfoil, the aerodynamic centre is located at $25 \%$ of the chord length, $c$ from the leading edge according to Dowell et al. ${ }^{28}$ As a result, the aerodynamic moment is only dependent on lift force and the distance from aerodynamic centre to mounting axis, which leads to:

$$
\tau_{a}=L\left(x_{A C}-x_{M A}\right)
$$

(Equation 4)

Substituting Equations 2, 3 and 4 into Equation 1 leads to the characteristic equation of the system:

$$
L\left(x_{A C}-x_{M A}\right)=\kappa(\theta-\alpha)-\cos (\alpha)\left(x_{C o G}-x_{M A}\right) m_{w} g
$$

(Equation 5)

The wind velocity, $v$ according to angle of incidence for a fixed spring preload angle is of great interest for this work. It is derived by substituting the general lift equation into Equation 5 and re-arranging to show,

$$
\begin{gathered}
0.5 C_{L} \rho_{A i r} v^{2} s c\left(x_{A C}-x_{M A}\right)=\kappa(\theta-\alpha)-\cos (\alpha)\left(x_{C o G}-x_{M A}\right) m_{w} g \\
\Leftrightarrow v(\alpha)=\sqrt{\frac{\kappa(\theta-\alpha)-\cos (\alpha)\left(x_{C o G}-x_{M A}\right) m_{w} g}{0.5 C_{L} \rho_{A i r} s\left(x_{A C}-x_{M A}\right)}}
\end{gathered}
$$

(Equation 6)

where s and c are the span and chord of the airfoil respectively. 
According to two-dimensional thin-airfoil-theory, the lift coefficient $C_{l}$ increases linearly at low angles of incidence and is scaled theoretically by the factor $2 \pi,{ }^{10}$ so in terms of degrees, Equation 6 becomes,

$$
v(\alpha)=\sqrt{\frac{\kappa(\theta-\alpha)-\cos (\alpha)\left(x_{C o G}-x_{M A}\right) m_{w} g}{\alpha \frac{\pi^{2}}{180} \rho_{A i r} s c\left(x_{A C}-x_{M A}\right)}}
$$

(Equation 7)

Furthermore, a theoretical approximation for a finite wing based on literature is also deduced. With a three-dimensional wing, the flow can spill around the ends of the airfoil resulting in a pressure loss. The magnitude of this effect is dependent on the aspect ratio $(A R)$. A second approach to approximate the performance of a three-dimensional wing in freestream is taken into consideration. The calculation is borrowed from two-dimensional theory, but the lift coefficient is recalculated according to Katz, ${ }^{30}$ where $\alpha$ is in radians.

$$
C_{L, 3 D}=\frac{2 \pi \alpha}{1+\frac{2}{A R}}=2 \pi \alpha \eta_{K a t z}
$$

(Equation 8)

This correction suggested by Katz ${ }^{30}$ equals an aerodynamic efficiency factor of $\eta_{\text {Katz }}=53.27 \%$ for the given $A R=2.28$.

$$
v(\alpha)=\sqrt{\frac{\kappa(\theta-\alpha)-\cos (\alpha)\left(x_{C o G}-x_{M A}\right) m_{w} g}{\alpha \frac{\pi^{2}}{180} \eta_{K a t z} \rho_{A i r} s c\left(x_{A C}-x_{M A}\right)}}
$$

(Equation 9)

Equation 7 and 9 enables the comparison of two-dimensional aerodynamic theory and three-dimensional wing in freestream to that of the wind tunnel experiments reported later in this work. Note, that this theory can only be applied pre-stall and for incompressible fluids. A full mathematical model will include lift and drag for the complete system. 
However, in this proof of concept work, we only consider the moments in the derivation and validate this with wind tunnel experimentation.

\subsection{Experimental setup}

The standard NACA 0012 wing profile is used, which is based on coordinate points provided by the University of Illinois at Urbana-Champaign. ${ }^{31}$ A chord length, $c$ of $100 \mathrm{~mm}$ is used with a wingspan, $s$ of $228 \mathrm{~mm}$, resulting in an aspect ratio $A R=2.28$. The maximum thickness, $t$ of $12 \mathrm{~mm}$ is located at $30 \%$ chord. The wing is fitted with a hole for the mounting rod, which is located at $10 \%$ chord. We use a $3 \mathrm{D}$ printed wing with refined surfaces. The printing density is set to $25 \%$ fill rate using Acrylonitrile Butadiene Styrene (ABS). As a result, the wing is of a lightweight construction and the mass, $m_{w}$ is measured as $119.3 \mathrm{~g}$ and the centre of gravity determined to be at $42.4 \%$ chord.

The wing and spring system is mounted to a transparent box that accommodates the entire system. It is placed in the wind tunnel as a whole. The outer dimensions are $450 \mathrm{~mm} \mathrm{x}$ $262 \mathrm{~mm} \times 350 \mathrm{~mm}$. The box is made of lasered acrylic glass. Precise holes in the acrylic walls are used as a bearing for the mounting rod, which is inserted into the wing. Friction in the bearings is reduced by greasing them before all tests. The wing is locked to the mounting rod geometrically via a small removable pin. A recess is provided in the side of the wing and a hole is provided in the mounting rod. Once the wing is fitted on the mounting rod within the apparatus, the locking pin is inserted into the hole. Subsequently, the wing slides along the mounting rod, thus the locking pin is fitted into the recess. This allows a quick and toolless assembling and disassembling. Note that a small gap between the wing and the walls is needed for this mechanism. This gap measures approximately $10 \mathrm{~mm}$ on both sides. The gap allows flow from top surface to the bottom surface due to the pressure difference created. Therefore, the system cannot be treated as strictly quasi-twodimensional. A panel, which is mounted to the wall, accommodates the spring system. The spring system consists of a spiral spring, which is connected to the mounting rod and to a configurable mount on the transparent box. 36 notches in the mounting plate enable fixing positions of the spring in $10^{\circ}$ steps. In this way, the preload of the spring is selected. The initial angle of incidence is limited by a small pin that compensates the spring force. The pin, which we refer to as angle of incidence limiter, can be adjusted vertically, whereby the 
initial angle of incidence is selected. A degree scale is attached to the opposing wall, which allows for direct readings of angle of incidence.
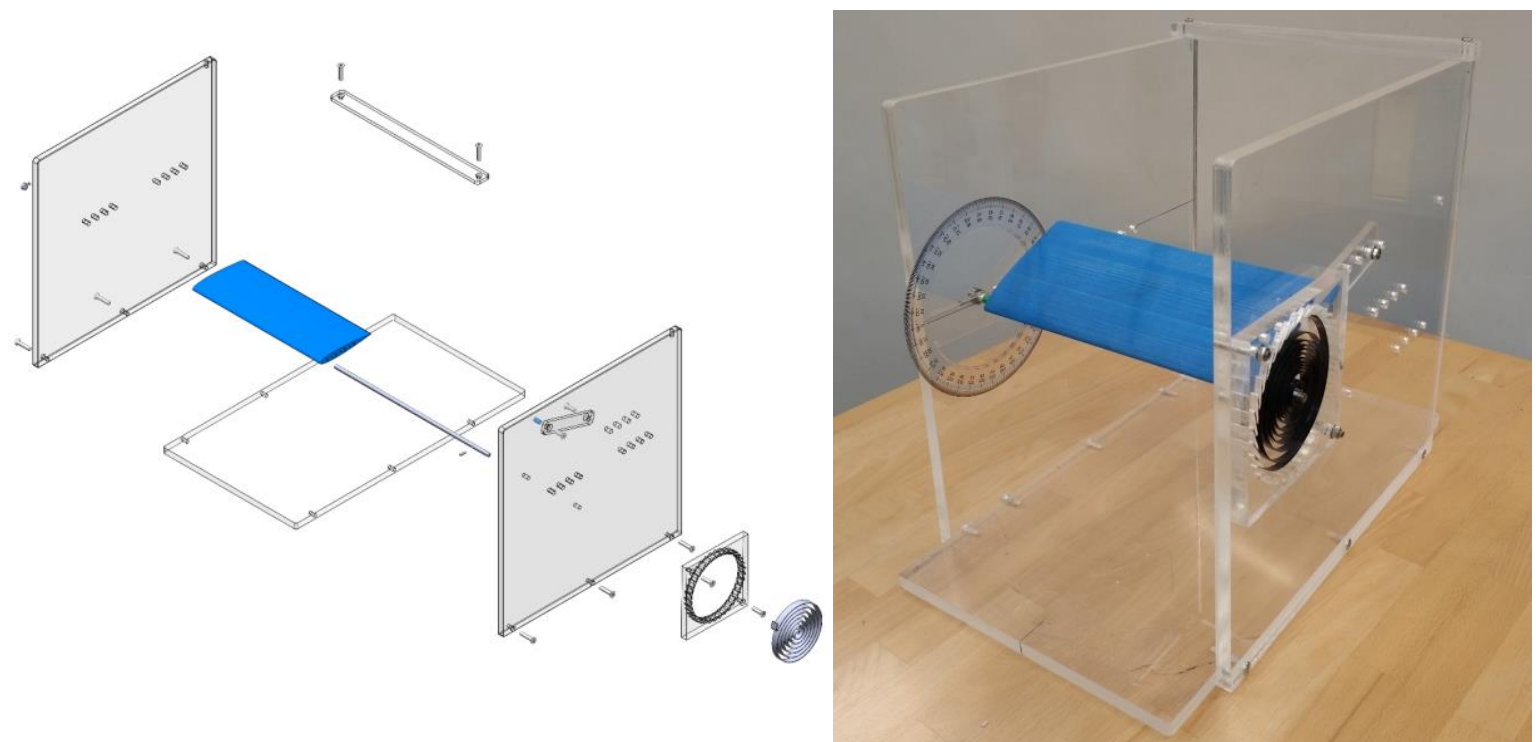

Figure 2) Experimental setup a) exploded view in CAD b) picture

The experiments are conducted in the University of Portsmouth's low-speed wind tunnel, which is open circuit with a closed test section. The maximum airflow velocity is $v_{\max }=$ $20 \mathrm{~m} / \mathrm{s}$ and reliable results can be obtained above $v_{\min }=4 \mathrm{~m} / \mathrm{s}$. Each measurement is taken once the airflow has stabilised and static equilibrium is reached. Considering also the operating limitations of the wind tunnel $v_{\min }$ and $v_{\max }$ the maximum and minimum Reynolds number based on chord length are respectively $R e_{\min }=2.7 \times 10^{4}$ and $R e_{\max }=1.36 \times 10^{5}$. Thence, laminar flow is expected throughout this work. A Furness Controls Ltd. FCO12 digital micro-manometer is used to record the flow velocity. The overall uncertainty of wind velocity measurement is $\pm 0.1 \mathrm{~m} / \mathrm{s}$ due to inhomogeneous flow in the wind tunnel. The test chamber has a cross-section of $455 \mathrm{~mm}$ high by $455 \mathrm{~mm}$ wide with chamfered corners. The usable space for test equipment is not only limited by chamber dimensions, but also by the hatch opening, which is $305 \mathrm{~mm}$ wide and $610 \mathrm{~mm}$ long. The cross-sectional area is $0.2 \mathrm{~m}^{2}$ and the blockage estimated to be $13.8 \%$ when the wing is at the starting position of $40^{\circ}$. At $10^{\circ}$ the blockage ratio reduces to $8.4 \%$. We note these blockage ratios are relatively large and would necessitate a correction factor to be 
truly representative to freestream conditions. However, the main objective of this work is proof of concept, so we neglect blockage corrections to aid simplicity.

Oscillation of the wing is expected when the angle of incidence of the wing is close to the stall angle, due to large scale vortices being shed. The frequency of these oscillations is captured by using high-speed video footage, which is taken at 240 frames per second. The videos are analysed using the open source software 'Tracker', which is based on the Open Source Physics Java Framework, to track the motion of the trailing edge of the wing. Both manual and automatic object tracking are used with identical results. To increase accuracy, data sets of 10 oscillations are measured, using time readings provided by the software. Furthermore, at least four readings are taken to ensure repeatability and to help determine whether the frequency is constant. It must be mentioned, that this technique only enables the identification and measurement of constant frequencies. This assumption of constant frequency is believed to hold in the current work, but would require further measurement to prove conclusively.

\section{Validation of system}

\subsection{Spring calibration}

Before conducting wind tunnel experiments, preliminary tests are conducted to assess the system, calibrate the spring and identify the torsional spring constant, $\kappa$. The experimental setup of this preliminary test is the same as used for the wind tunnel experiments reported later, except that a moment is applied mechanically by applying a weight force at the trailing edge, instead of aerodynamically.

The spring is calibrated by identifying the zero point. Therefore the spring is loaded starting at the lowest preload to compensate for the gravitational force of the wing and to overcome friction reliably. The spring preload is then raised incrementally in steps of $40^{\circ}$. The results are fitted by a linear function in Python and extrapolated. From this, numerical values of both the torsional spring constant and the zero point of the angle can be derived. The gradient of the linear function and hence the torsional spring constant is $\kappa=$ $0.1638 \mathrm{Nmm} / \mathrm{deg}$. The uncertainty of the fitting is $\pm 0.0032 \mathrm{Nmm} / \mathrm{deg}$ for the torsional spring constant with a linear spring deformation. 
We also define the required aerodynamic moment for an equilibrium state at zero angle of incidence in Equation 10, which serves as a reference for the applied spring preload throughout this work. This is more relevant than specifying the spring torque only and a sufficient approximation of Equation 5 for small angle of incidence. We name it torque setting $\tau_{a, \alpha=0}$.

$$
\tau_{a, \alpha=0}(\theta)=\kappa \theta-\left(x_{C o G}-x_{M A}\right) m_{w} g=L\left(x_{A C}-x_{M A}\right)=\left(c-x_{M A}\right) m_{s} g
$$

(Equation 10)

For the symmetrical NACA 0012 airfoil, the required aerodynamic moment is theoretical as no lift is achieved at zero degrees. Nevertheless, we elect to use the zero degrees to be the most suitable reference in this work. For cambered airfoils an aerodynamic moment is achievable at zero degrees. As can be seen in Equation 10, the required aerodynamic moment is equal to a lift acting at the aerodynamic centre with wind velocity, which is also equal to a weight $m_{s}$ acting at the trailing edge with no wind velocity.

\subsection{Application of aeroelastic theory and comparison to experiment}

Experimental findings and results derived from aeroelastic theory are compared for a fixed torque setting. This section focuses solely on low angles of incidence in the suspected prestall region. Theoretical behaviour is calculated for an idealised two-dimensional airfoil and a finite wing with end effects. The behaviour of the system is demonstrated according to an increasing airflow velocity, while the angle of incidence adapts to it. An invariable spring preload angle of $\theta=456.7^{\circ}$ is considered, which corresponds to a required aerodynamic moment at zero degrees of $\tau_{a, \alpha=0}=36.92 \mathrm{Nmm}$. At each measurement point, the system is in a static equilibrium of aerodynamic, spring and gravitational forces. The results for the two-dimensional idealised infinite wing model, the three-dimensional finite wing model and the data including uncertainty from the wind tunnel experiment are plotted in Figure 3. The estimated error in reading the angle is $+/-0.5$ degree. The uncertainty in the wind velocity is $+/-0.1 \mathrm{~m} / \mathrm{s}$ resulting in an estimated error of Reynolds number to be $+/$ 700. This uncertainty is consistent throughout this work for all experimental measurements, however we only include it in Figure 3 to aid clarity. 


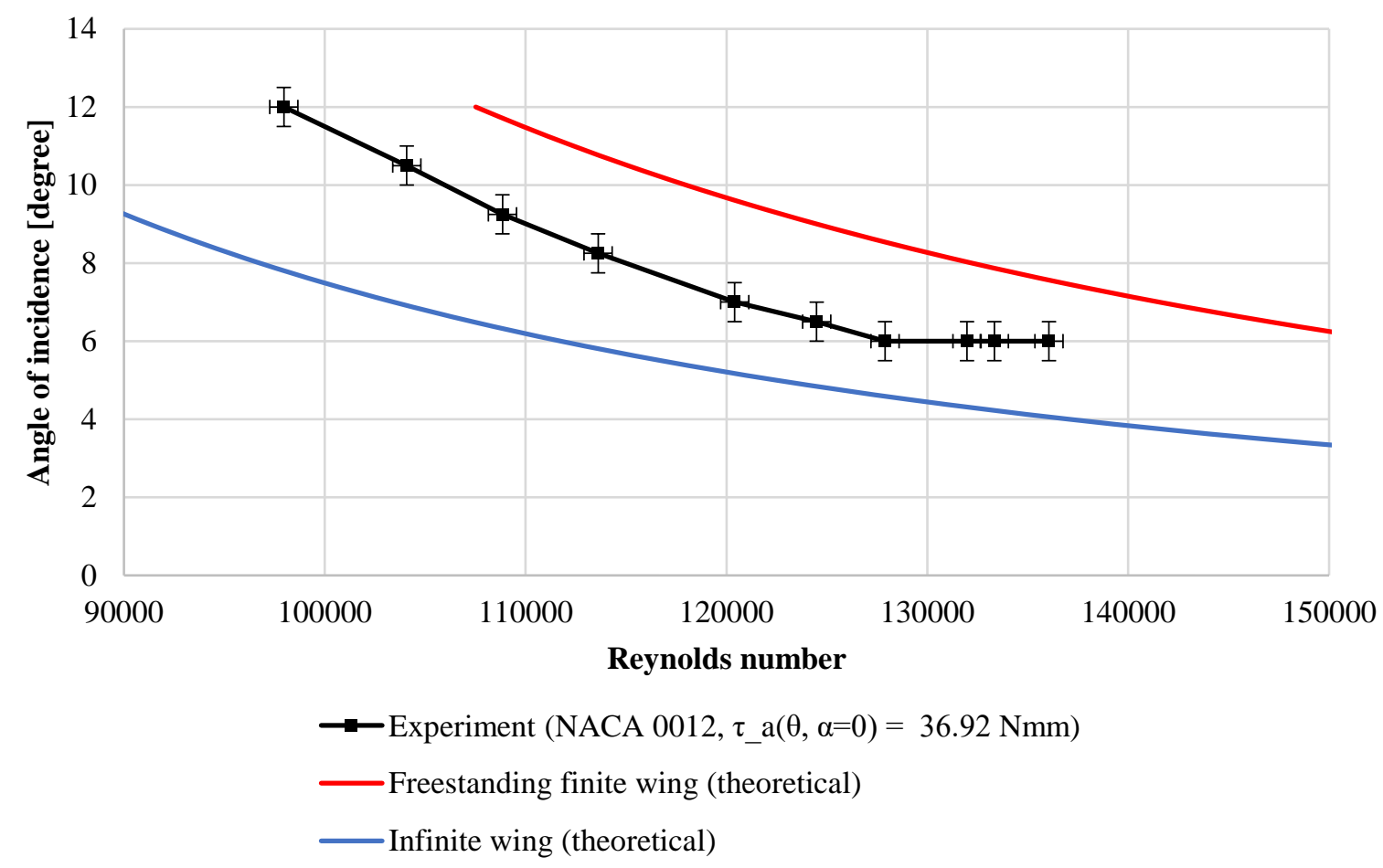

Figure 3) Comparison of experimental and theoretical angle of incidence response to flow velocity with finite and infinite wings.

Compared to the two-dimensional theory, higher velocities are necessary to rotate the wing in the wind tunnel experiment. Thus, the actual aerodynamic moment is as expected less than the aerodynamic moment derived from two-dimensional thin-airfoil-theory. However, it is also observed, that the wing generates more lift than suggested by the approximation of a three-dimensional finite wing. Therefore, the current experimental setup responds within expected limits. The wind tunnel experiments prove the basic principal of adapting the angle of incidence according to flow velocity.

\section{Results and Discussion}

\subsection{General operation of the aeroelastic system, pre-, near- and post-stall}

Having validated our system in low angles of incidence in the pre-stall region, we now look at angles beyond the stall region and up to $40^{\circ}$. Figure 4 presents the results of a typical wind tunnel test at an unchanged torque setting of $\tau_{a, \alpha=0}=36.92 \mathrm{Nmm}$. The angle 
of incidence is mechanically limited by the angle of incidence limiter to a maximum of $40^{\circ}$, which we use as a starting point. The moment, which the wing has to overcome initially to rotate trailing edge down, is resulting from Equation 5 and is $39.23 \mathrm{Nmm}$ respectively. At a wind velocity of about $8 \mathrm{~m} / \mathrm{s}$ in this example, the first movement of the wing is observed as can be seen in Figure 4. With further increasing flow velocity, the wing rotates and consequently the angle of incidence decreases.

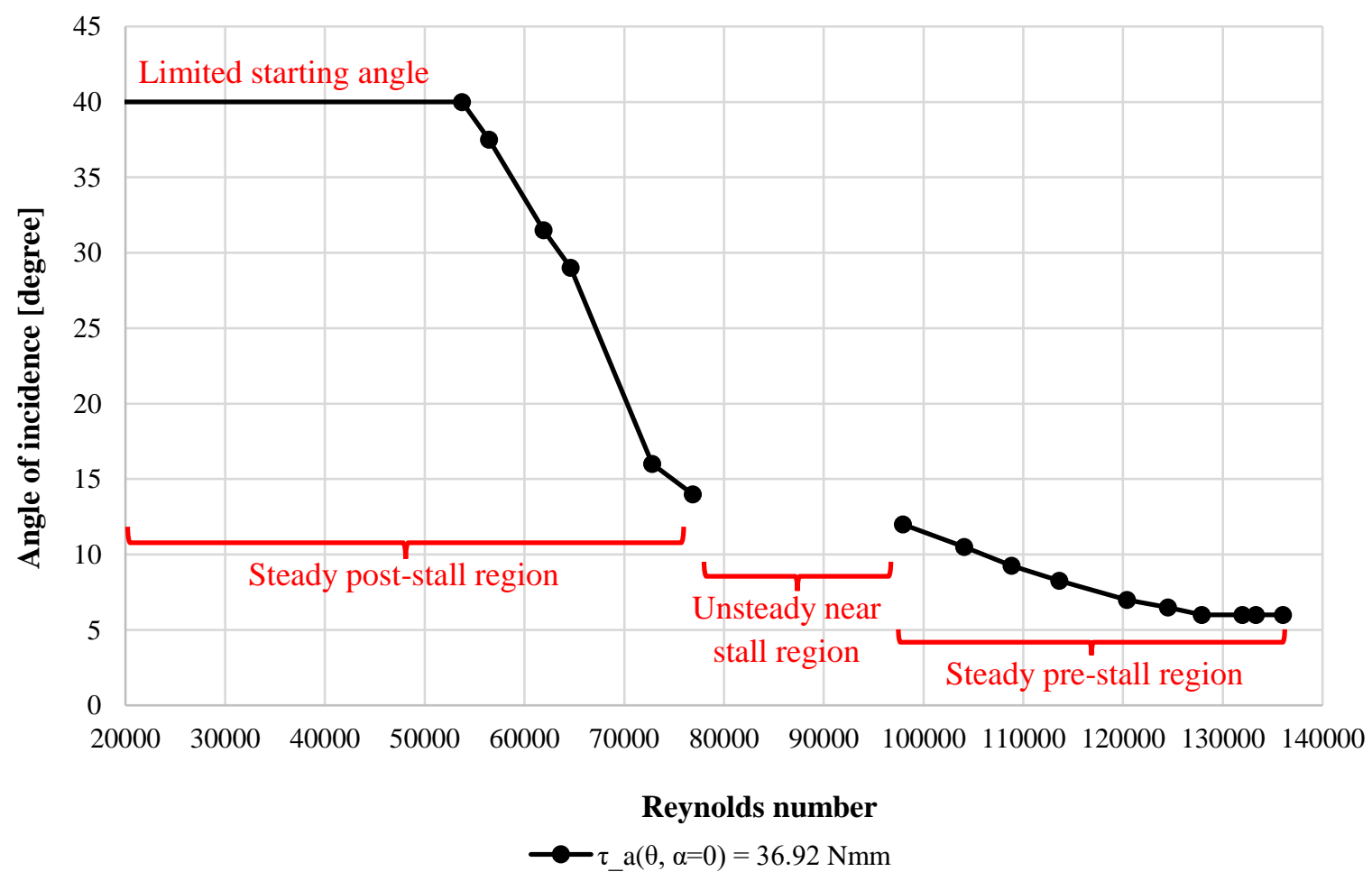

Figure 4) Three regions of a typical angle of incidence response to airflow velocity.

In this example, at wind velocities between 8 and $11 \mathrm{~m} / \mathrm{s}$, the angles of incidence are all above $15^{\circ}$ and the wing is stalled but exhibits steady behaviour. At wind velocities between 12 and 14m/s, the wing exhibits unsteady behaviour and is in the near-stall region. At wind velocities above $15 \mathrm{~m} / \mathrm{s}$, the angle of incidence is below $12^{\circ}$ and the wing again exhibits steady behaviour. Thence, the angle of incidence response to airflow velocity is divided into three regions according to Figure 4. For angles of incidence less than the critical stall angle, the behaviour is designated as pre-stall. ${ }^{32}$ At the present Reynolds numbers, the stalling point is expected to be at an angle of incidence of about $12^{\circ},{ }^{23}$ which correlates well with our work. The gap between steady post-stall and steady pre-stall 
region is dominated by unsteady behaviour of the wing in this near-stall region. Data points are shown in Figure 4 and connected to indicate stable behaviour, while unstable behaviour is identified by a gap to visualise the certain flow velocities at which unsteady behaviour occurs.

\subsection{Repeatability of results}

The repeatability of results is essential for confidence of the experimental setup and measurement methods, but also a basic prerequisite for any automotive application. The results presented in Figure 5 are obtained from four separate wind tunnel experiments for the same torque setting. The overall deviation is satisfactorily small as can be seen in Figure 5. However, in the post-stall region, the spread is slightly more pronounced. Nevertheless, the results show good agreement on the whole and are deemed sufficient for our purpose.

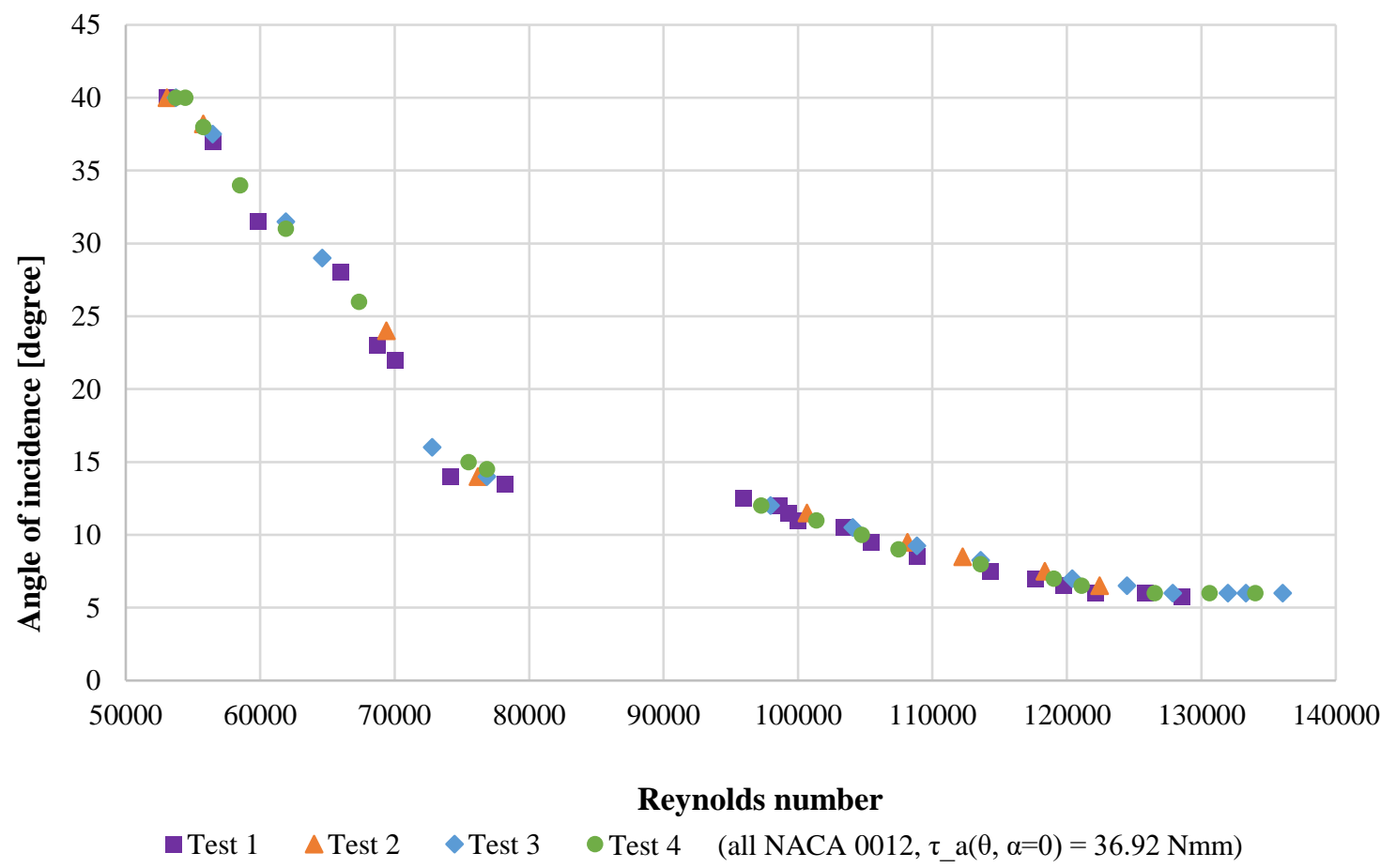

Figure 5) Superposition of four independent wind tunnel experiments with identical setup to demonstrate repeatability. 


\subsection{Impact of spring preload}

The results for various spring preload settings with increasing flow velocities are presented in Figure 6. The spiral spring is adjusted to settings from $\theta=296.7^{\circ}$ to $\theta=536.7^{\circ}$ in incremental steps of $40^{\circ}$, resulting in torque setting within a range of 10.71 to $50.02 \mathrm{Nmm}$. Starting at the limit of $40^{\circ}$ again, the angle of incidence is decreasing. The general shape of the response remains similar for all torque settings but is shifted towards higher velocities for higher spring preloads. The gradient of post-stall response however is slightly decreasing. Furthermore, the final angle of incidence, at which the wing did not rotate any further despite increasing velocity, is also increasing with higher spring preload. The prestall response mode is parabolic in shape for all torque settings. Oscillatory behaviour of the wing is found for all spring preloads except for the lowest torque setting tested.

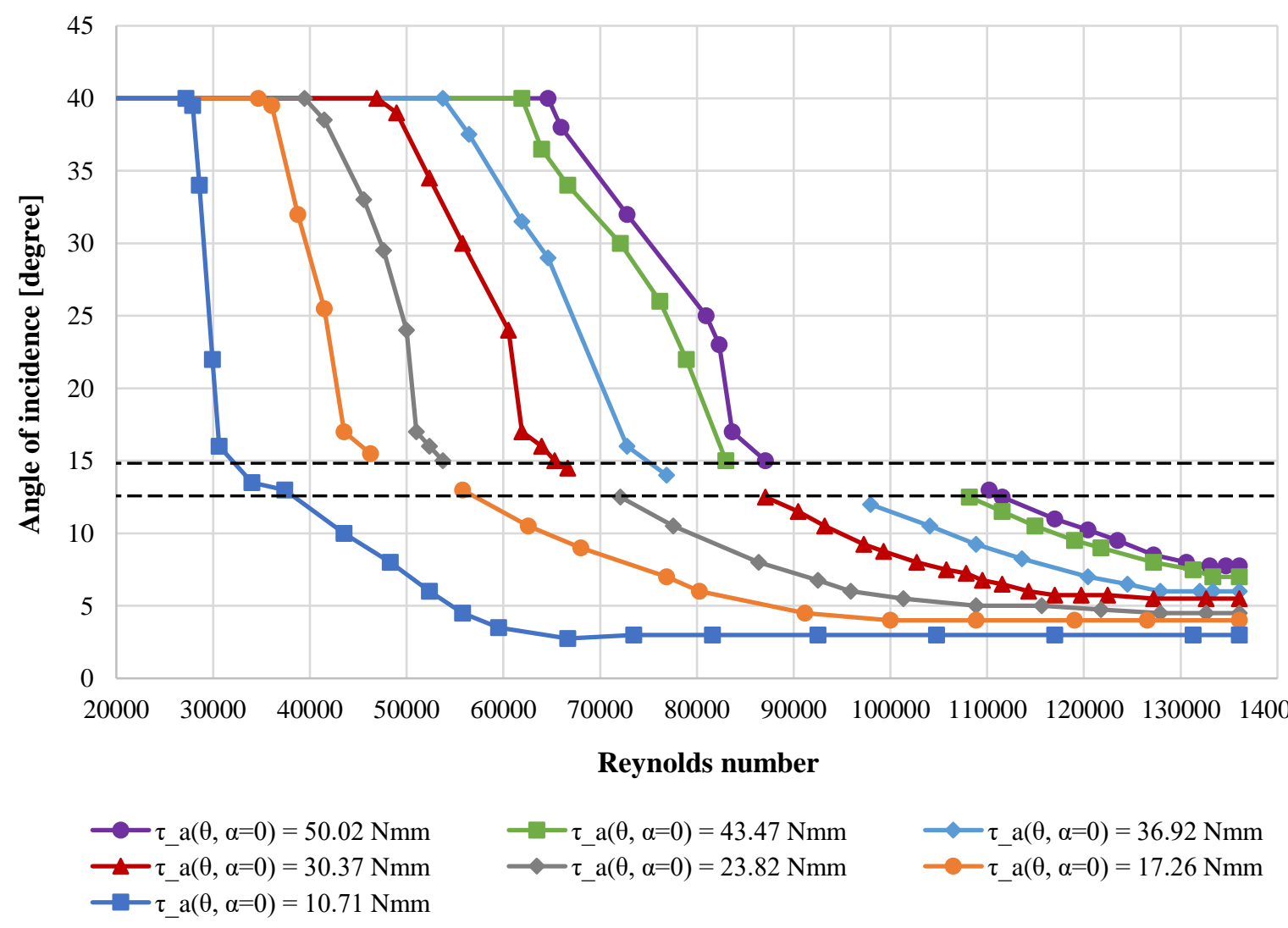

Figure 6) Angle of incidence response for various spring preloads of the NACA 0012 wing with boundary of unsteady angles. 
The largest variations in angles of incidence with wind velocity can be seen by the steep gradients in Figure 6. These occur in the pre-stall regions using lower velocities. Typically a $25 \%$ increase in wind velocity corresponds to a reduction in angle of incidence from $40^{\circ}$ to $20^{\circ}$. The gradients are lower in the pre-stall region using higher velocities. Here a typical increase of $25 \%$ in wind velocity corresponds to a reduction in angle of incidence from $12^{\circ}$ to $7^{\circ}$. A further comparison with theory using finite and infinite wings with the various torque settings in the pre-stall region is shown in Figure 7. Two-dimensional infinite wing theoretical results are represented by dotted lines, whereas the theoretical responses of free-standing finite wings are shown with continuous lines.

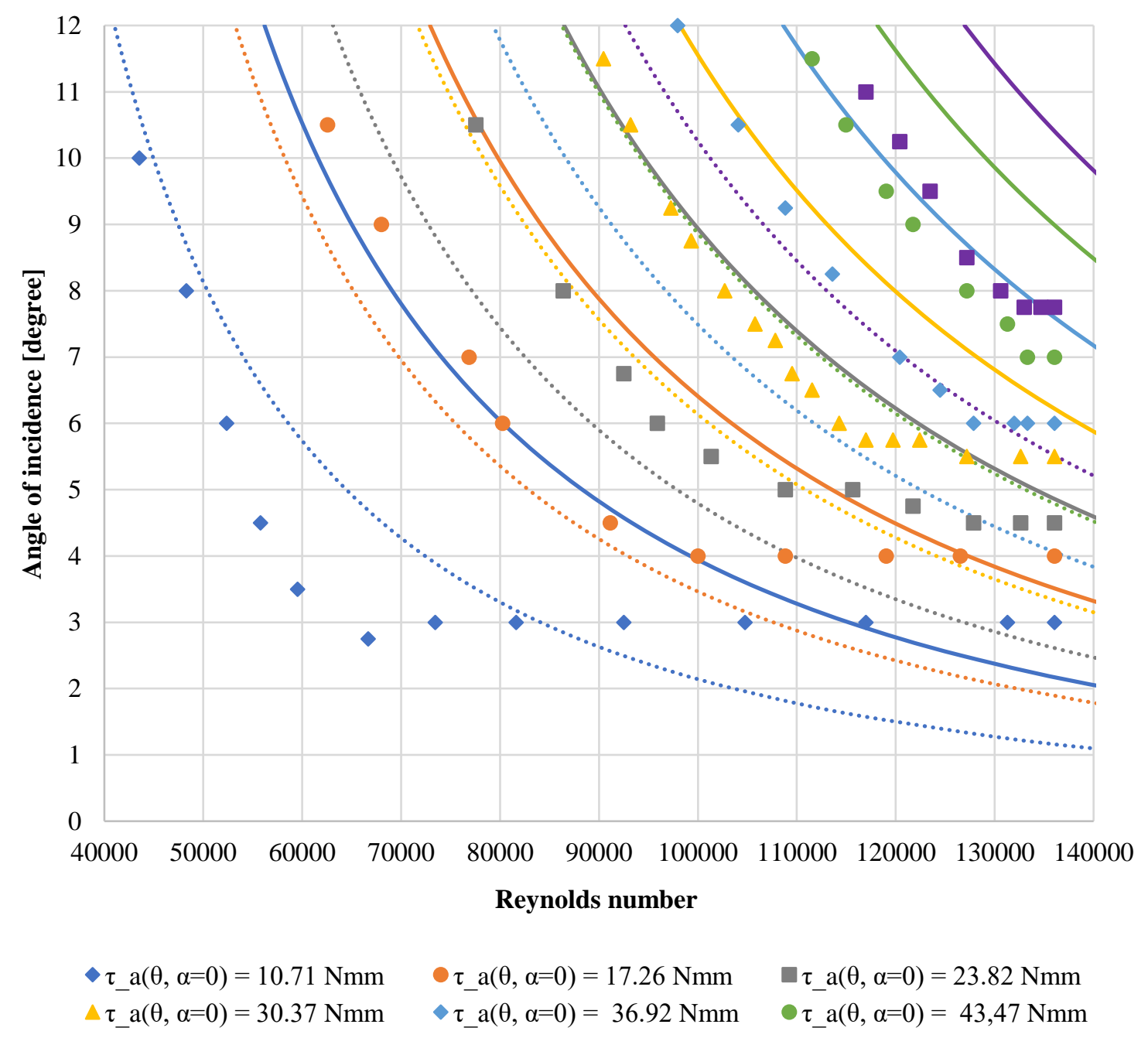

Figure 7) Comparison of experimental data (dots), 2D-theory (dotted) and 3D-theory (continuous) in corresponding colours. 
The experimental results in Figure 7 are expected to be in between the dotted and continuous lines for all torque settings. However, this is not the case for all measurement points using the lowest 2 spring preload settings. Most noticeable is, that the wind tunnel result exceeds the two-dimensional theory at the lowest torque setting of $\tau_{a, \alpha=0}=$ $10.71 \mathrm{Nmm}$. We suspect this is due to friction effects not being overcome, but further analysis is required to prove conclusively.

It can be seen in Figure 7, using either theory or experimental results, that the angle of incidence is decreasing with wind velocity. The coefficient of lift and also coefficient of drag are also therefore decreasing. However, due to the increase in wind velocity the lift remains near constant at these low angles. Likewise, the drag would also increase with higher wind velocities but in the case of a spring mounted wing, this increase is reduced due to the lower angle of incidence obtained. Tables 1 and 2 give an example of changes in drag and lift using $6^{\circ}$ and $40^{\circ}$ and comparing with $4^{\circ}$ in the experiment using a torque setting of $\tau_{a, \alpha=0}=17.26 \mathrm{Nmm}$.

Table 1) Example comparison of data for fixed wing at $14.7 \mathrm{~m} / \mathrm{s}$ and compared to spring mounted wing.

\begin{tabular}{ccccccc}
\hline Angle (deg.) & $C_{L}$ & $C_{D}$ & Lift $(\mathrm{N})$ & Drag $(\mathrm{N})$ & \% reduction in lift & \% reduction in drag \\
\hline 4 & 0.44 & 0.0124 & 1.3289 & 0.0375 & 0 & 0 \\
6 & 0.66 & 0.0152 & 1.9933 & 0.0459 & 33.3 & 18.4 \\
40 & 1.075 & 0.92 & 3.2466 & 2.7785 & 59.1 & 98.6 \\
\hline
\end{tabular}

Table 2) Actual values of lift and drag for spring mounted wing at various wind velocities and compared to fixed.

\begin{tabular}{cccccccc}
\hline $\begin{array}{c}\text { Angle } \\
(\text { deg. })\end{array}$ & $\begin{array}{c}\mathrm{v} \\
(\mathrm{m} / \mathrm{s})\end{array}$ & $\begin{array}{c}\text { Lift } \\
(\mathrm{N})\end{array}$ & $\begin{array}{c}\text { Drag } \\
(\mathrm{N})\end{array}$ & $\begin{array}{c}\text { actual \% } \\
\text { increase in lift }\end{array}$ & $\begin{array}{c}\text { actual \% } \\
\text { increase in drag }\end{array}$ & $\begin{array}{c}\% \text { diff. } \\
\text { in lift }\end{array}$ & $\begin{array}{c}\% \text { diff. in } \\
\text { drag }\end{array}$ \\
\hline 4 & 14.7 & 1.3289 & 0.0375 & 0 & 0 & 0 & 0 \\
6 & 11.8 & 1.2844 & 0.0296 & 3.46 & 26.6 & 55.2 & 55.2 \\
40 & 5.3 & 0.4220 & 0.3612 & 214 & -89.6 & 669 & 669 \\
\hline
\end{tabular}

Using approximate lift and drag coefficients of 0.66 and 0.0152 respectively at $6^{\circ}$ and 0.44 and 0.0124 respectively at $4^{\circ}$ taken from Jain ${ }^{33}$ would correlate to reductions of $33 \%$ and 
$18 \%$ in lift and drag coefficients respectively. Using ISA conditions, this would correlate to a lift of $1.28 \mathrm{~N}$ and drag of $0.03 \mathrm{~N}$ at $6^{\circ}$ and $11.8 \mathrm{~m} / \mathrm{s}$ and $1.33 \mathrm{~N}$ of lift and $0.037 \mathrm{~N}$ of drag at $4^{\circ}$ and $14.7 \mathrm{~m} / \mathrm{s}$. Therefore a $3 \%$ increase in lift and $27 \%$ increase in drag. Over the same velocity range, a fixed wing at $6^{\circ}$ would show an increase in lift and drag of $55 \%$. Thence, a reduction in drag of $18 \%$ using spring mounted wing over a fixed wing is found.

Incidentally, these experimental points used also lie on the 3D finite wing curve in Figure 7 with the torque setting of $10.71 \mathrm{Nmm}$, so that the same results are found for lift and drag. It can be concluded that within a particular range, the spring mounted wing allows for near constant lift and reduced drag when compared with a fixed wing when increasing wind velocity. This is further illustrated in Figure 8 where the lift and drag are plotted against Reynolds number which varies only due to wind velocity.

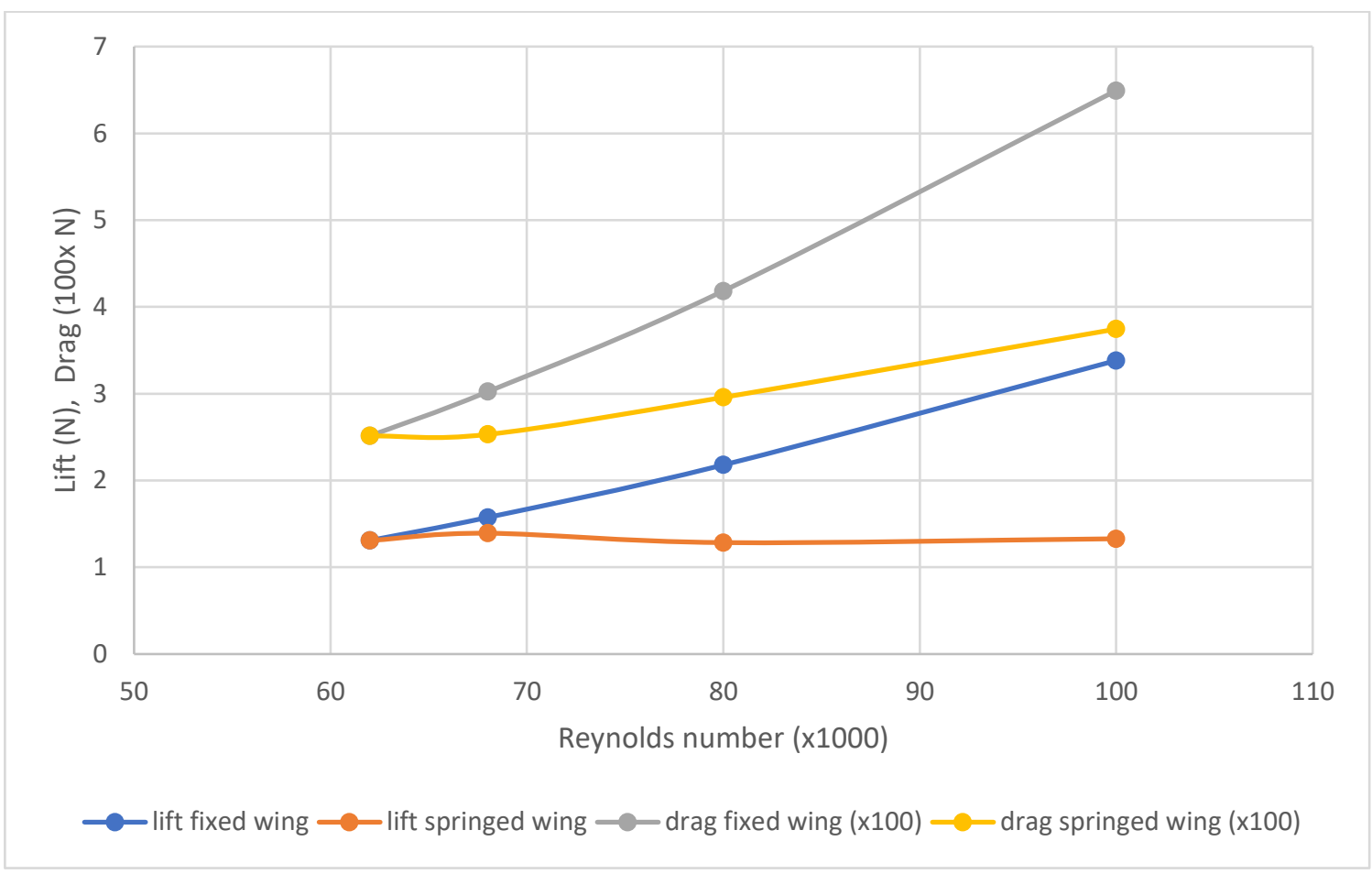

Figure 8) Comparison of lift and drag for fixed wing and spring mounted wing starting from 10.5 degrees with varying Reynolds number in pre-stall region.

The lift and drag increase according to the parabolic relationship with velocity for the fixed wing as expected. The lift on the spring mounted wing can be seen to hold relatively constant over the Reynolds number range shown. However, the drag is shown to increase but is much smaller increase than the fixed wing. This reduction of approximately $43 \%$ 
would result in a lower power requirement of the same or generate a higher top speed being achievable of the order of $19 \%$ according to Barnard ${ }^{5}$. It should be noted that the cornering speed would be reduced due to the reduced downforce obtained when compared to a fixed wing. Care must be taken to ensure the spring is kept at its initial position until the velocity of the car exceeds that of the highest speed in a corner so as not to loose downforce in the corner. Hence, the initial starting position and spring stiffness need to be tailored for each circuit.

Higher reductions in angles of incidence and corresponding drag coefficients are possible when going through the stall region, but this would incur transient effects. Using the same torque setting of $\tau_{a, \alpha=0}=17.26 \mathrm{Nmm}$ in the experiment and approximate lift and drag coefficients of 1.075 and 0.92 respectively at $40^{\circ}$ would correlate to reductions of $59 \%$ and $98 \%$ in lift and drag coefficients respectively when compared with $4^{\circ}$. Using ISA conditions, this would correlate to a lift of $0.42 \mathrm{~N}$ and drag of $0.36 \mathrm{~N}$ at $40^{\circ}$ and $5.3 \mathrm{~m} / \mathrm{s}$. Therefore a $215 \%$ increase in lift and $90 \%$ reduction in drag when compared with $4^{\circ}$. Over the same velocity range, a fixed wing at $40^{\circ}$ would show an increase in lift and drag of $670 \%$. Thence, a reduction in drag of $98 \%$ using spring mounted wing over a fixed wing is found. It can be concluded that within a particular range, a larger variation in wind velocity and corresponding change in angle of incidence will correspond to greater drag reductions and efficiencies. Although, transient effects could have a severe detrimental impact.

\subsection{Unsteady aeroelastic behaviour}

Unsteady behaviour of aerodynamic devices is a highly adverse phenomenon in most applications and especially in race car aerodynamics. Oscillation about the rotational axis is a potential threat for applicability of the proposed system. Indeed, a self-sustained oscillation is observed at all torque setting except for $\tau_{a, \alpha=0}=10.71 \mathrm{Nmm}$, which is the lowest setting in this experiment.

The oscillatory behaviour of the setup, which is specified at selected spring preload angels of $\theta=336.7^{\circ}$ to $\theta=536.7^{\circ}$, respectively $\tau_{a, \alpha=0}=17.26 \mathrm{Nmm}$ to $\tau_{a, \alpha=0}=50.02 \mathrm{Nmm}$, is further investigated. The oscillation is first overserved at an angle of incidence of about 
$15^{\circ}$ and ceased below a threshold of about $12^{\circ}$ to $13^{\circ}$. This is observed analogically for all torque settings and corresponding velocities. The Reynolds numbers of the examined measurement points range from $4.6 \times 10^{4}$ up to $1.1 \times 10^{5}$.

To explain this phenomenon, the stalling characteristics of the NACA 0012 wing, which are dependent on Reynolds number, need to be considered. It is well known, that at an angle of incidence higher than the stalling angle, the airflow separates from the wing. The observed oscillation is settled at an angle of incidence slightly above the expected stalling angle. However, compared to stalling characteristics of a fixed wing, the present system is more complex.

It is speculated, that the aeroelastic phenomenon of dynamic stall is detected. Dynamic stall in general is an unsteady aerodynamic effect, which is dominated by airflow alternating between attachment and separation. This hypothesis is strongly suggested by comparison to other published literature. ${ }^{20-22}$ Although, the existence of a laminar separation bubble (LSB) cannot be proven with the current experimental setup, it is known, that separation and reattachment of a LSB could be the cause of the oscillation. ${ }^{23}$ Indeed, the oscillation is not observed at angles of incidence above $15^{\circ}$.

The absence of oscillation at the lowest spring preload $\left(\tau_{a, \alpha=0}=10.71 \mathrm{Nmm}\right)$ as shown in Figure 6, could possibly indicate a separation of airflow without a laminar reattachment to the wing. The Reynolds number at the critical angle of incidence is at $3 \times 10^{3}$ to $3.7 \times 10^{3}$ and therefore below the limit of oscillation proposed by Poirel et al. ${ }^{25}$ However, it must be mentioned, that this absence of oscillation could also be caused by not overcoming friction of the bearings at this low airflow velocity. The oscillation is further examined by utilisation of high-speed video footage. Figure 9 show a sample of images using $\tau_{\mathrm{a}, \alpha=0}=$ $36.92 \mathrm{Nmm}$ and a Reynolds number of $7.79 \times 10^{4}$. 


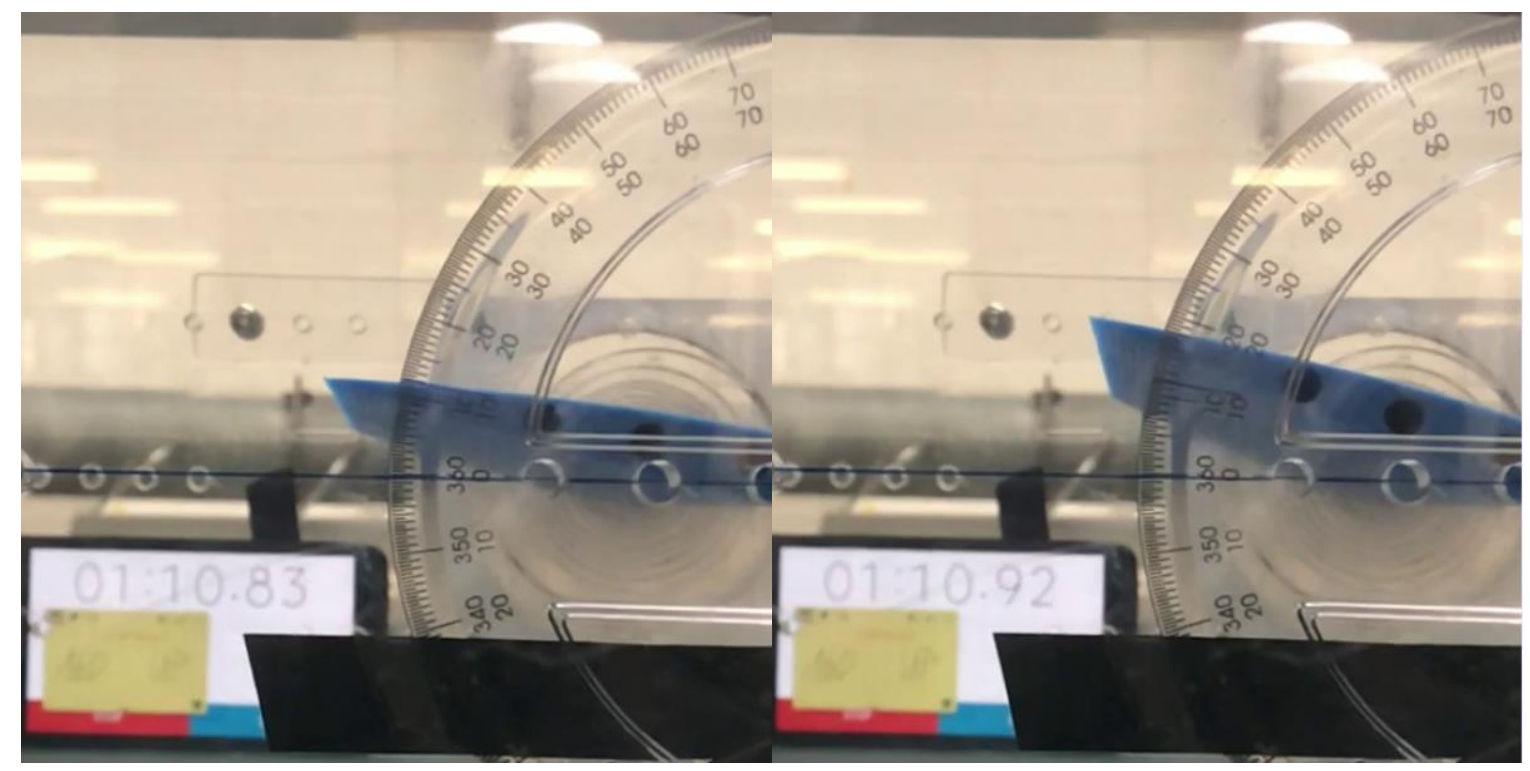

Figure 9) Sample of images at same wind velocity showing variation of incidence extremes in near stall region

The frequency of the symmetrical wing is found to be constant for a fixed flow velocity. The maximum deviation is less than 3\% from the average for all measured frequencies within the measurement time span of 15 to 45 seconds. The reduced frequency $K$ is defined as

$$
K=\frac{f c \pi}{v}
$$

(Equation 11)

where, $f$ is the measured frequency. This dimensionless frequency is used to provide a measure of unsteadiness and also allow for comparison of the oscillation to results from other experiments with different dimensions and airflow velocities. The torque setting $\tau_{a, \alpha=0}$ is used to indicate the incrementally increased spring preload and plotted with the corresponding Reynolds number to show the interaction of these three parameters, which is illustrated in Figure 10. 


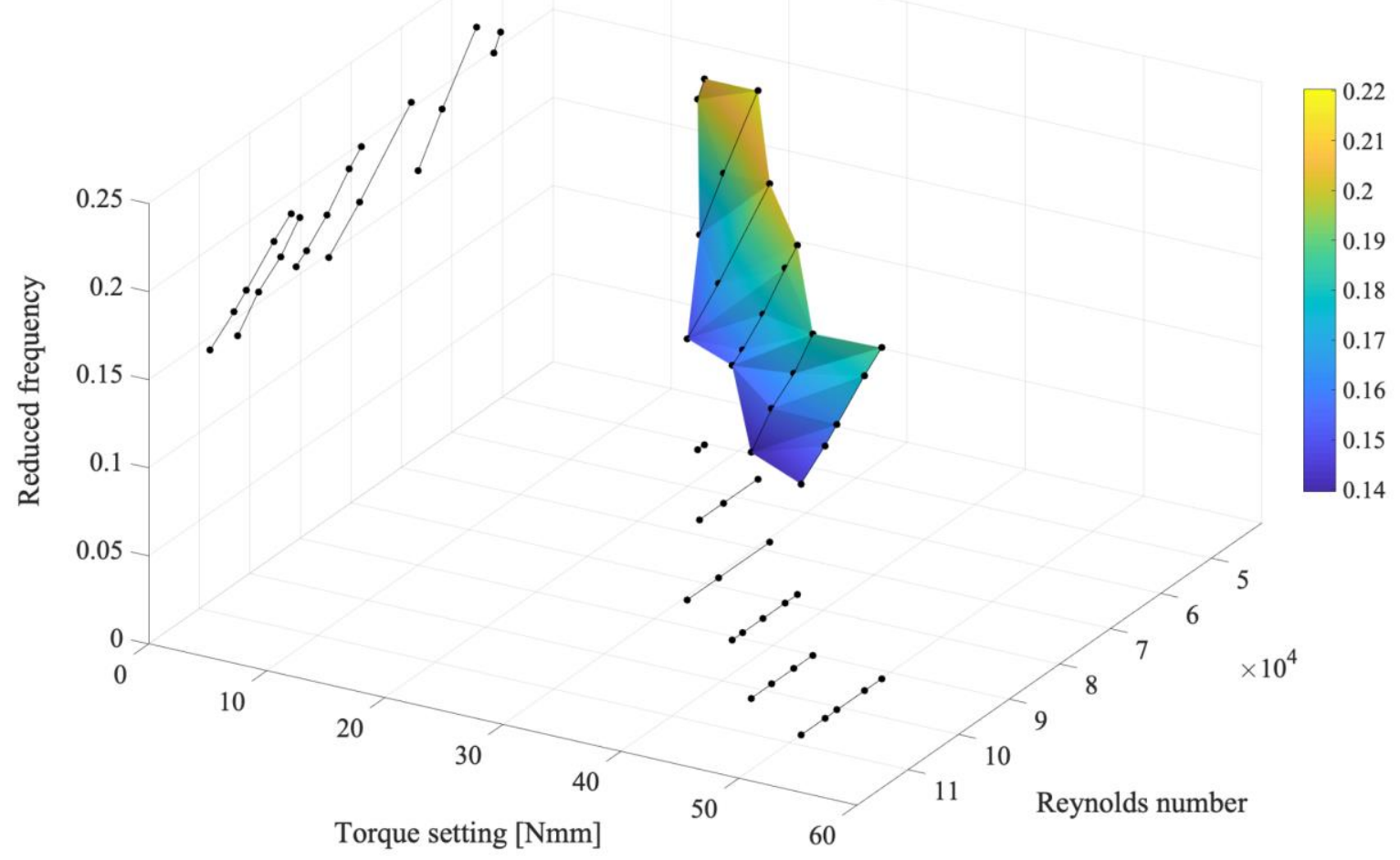

Figure 10) Plot showing unsteadiness region with reduced frequency values of the NACA0012 wing at various Reynolds numbers and spring preloads given as torque setting.

The results show, that the peak of unsteadiness is at $\tau_{\mathrm{a}, \alpha=0}=23.82 \mathrm{Nmm}$ and a Reynolds number of $5.51 \times 10^{4}$. A deceasing trend is observed both towards high Reynold numbers as well as high spring preload setting. Reduced frequencies are observed at minimum of 0.14 and a maximum of 0.22 . All observed reduced frequencies are very high, thus, the oscillation is considered highly unsteady in general. Similar reduced frequencies were observed by Poirel et al. ${ }^{25}$ at a range of 0.1 to 0.2 . The simple solution to avoid the unsteadiness and oscillations is to set the angle of incidence limiter to an angle of incidence of $12^{\circ}$ or less. 


\section{Evaluation and Conclusion}

A novel concept for an adaptive and passive wing based on an adjustable elastic element is successfully investigated experimentally. The general functioning of the proposed concept is verified, while challenges and limitations of the system are also encountered. The mechanism can be triggered at a configurable flow velocity. The comparison to experimental results reveal, that three-dimensional aerodynamic effects play a significant role. The knowledge of the location of the aerodynamic centre, lift coefficient and pitching moment coefficient are considered crucial for the success of a reliable prediction and adjustment of the system.

Oscillation of the symmetrical wing closely above the typical stalling angle is found to be periodic. Although not proven directly, strong arguments for oscillation to be caused by dynamic stall are presented. The behaviour is rated highly unsteady and is considered a major threat regarding the intended automotive or motorsport application. An operation is generally recommended only at pre-stall angle of incidence including a safety margin to provide consistent steady results.

It can be concluded, that the proposed concept is functional within aforementioned limitations. It must be mentioned, that the wind tunnel experiments are conduct at lower Reynolds numbers than expected in motorsport applications such as a rear wing. Experiments in a larger wind tunnel facility capable of higher flow velocities, so that wings with turbulent boundary layers can be fully understood, would be beneficial, in addition to directly measuring downforce and drag. The use of a cambered wings and at various mounting axis locations are of particular interest to enhance performance, but the effect of non-uniform flow on the system should be identified to provide accurate results applicable to road vehicles. The use of a full scale wind tunnel would assist as well as track testing, which would also allow the feel of the driver to be taken into account. Future work could also aim at addressing the periodic oscillation of the wing by attaching a damper to the system. In addition, the use of a variable spring rate to tailor the response to a specific requirement or race track is of potential interest. Future work could also include the effect of accelerations in all three directions, which could be combined with varying centre of 
gravity to add inertia effects, which could offer benefits under braking and acceleration as well as cornering.

\section{References}

1. Knight J, Spicak M, Kuzenko A, et al. Investigation of vehicle ride height and diffuser ramp angle on downforce and efficiency. Proc. IMechE, Part D: J. Automobile Engineering, 2018, DOI: 10.1177/0954407018776767

2. Hucho WH. Aerodynamics of road vehicles. $4^{\text {th }}$ ed. Warrendale, Pennsylvania: SAE International, 1998, p.391.

3. Bargende M, Reuss H-C and Wiedemann J. 14th Internationales Stuttgarter Symposium.1st ed. Springer, 2014, pp.521-531.

4. Reichelt S. Digital Transformer: Mercedes-Benz Concept IAA, http://icd.unistuttgart.de/?p=14605 (2017, accessed $7^{\text {th }}$ May 2019).

5. Barnard RH. Road vehicle aerodynamics. 3rd ed. Mechaero Publishing, 2010.

6. McBeath S. Competition Car Aerodynamics. 3rd ed. Poundbury, Dorchester, Dorset: Veloce Publishing Ltd, 2017.

7. Federation Internationale de l'Automobile. Regulations, https://www.fia.com/regulation/category/110 (2017, accessed $4^{\text {th }}$ May 2019).

8. Koenigsegg. The Regara - A new era, https://www.koenigsegg.com/regera (2017, accessed $9^{\text {th }}$ May 2019).

9. McLaren. Unthinkable Solutions, http://cars.mclaren.com/ultimate-series/mclarensenna/innovation (2017, accessed $9^{\text {th }}$ May 2018).

10. Fung YC. An Introduction to the Theory of Aeroelasticity. University of California, San Diego: Dover Publications, Inc. 1993.

11. Stodieck O, Cooper J, et al. Optimization of Tow-Steered Composite Wing Laminates for Aeroelastic Tailoring. AIAA Journal, 2015; 53(8): 2203-2215.

12. Dillinger JTS, Klemmik T, et al. Stiffness Optimization of Composite Wings with Aeroelastic Constraints. Journal of Aircraft, 2013; 50(4): 1159-1168.

13. De Breuker R, Binder S and Wildschek A. Combined Active and Passive Loads Alleviation through Aeroelastic Tailoring and Control Surface/Control System Optimization. In: AIAA Aerospace Sciences Meeting, Kissimmee, Florida: 8-12, 
2018, paper no. AIAA 2018-0764.

14. Weisshaar A and Duke DK. Induced Drag Reduction Using Aeroelastic Tailoring with Adaptive Control Surfaces, J Aircraft 2006; 43(1): 157-164.

15. Ajaj R, Friswell MD, et al. Performance and control optimisations using the adaptive torsion wing. The Aeronautical Journal 2012; 116(1184): 1061-1077.

16. Stanford B, Ifju P, et al. Fixed Membrane Wings for Micro Air Vehicles: Experimental Characterization, Numerical Modeling, and Tailoring. Progress in Aerospace Science, 2008; 44: 258-294.

17. Scott S, Capuzzi M, et al. Effects of aeroelastic tailoring on performance characteristics of wind turbine systems. Renewable Energy 2017; 114: 887-903.

18. Thuwis GA, De Breuker R, et al. Aeroelastic tailoring using lamination parameters - Drag reduction of a Formula One rear wing. Structural and Multidisciplinary Optimization 2010; 41(4): 637-646.

19. Ghommem M, Abdelkefi A, et al. Aeroelastic analysis and nonlinear dynamics of an elastically mounted wing. Journal of Sound and Vibration 2012; 331(26): 57745787.

20. McCrokskey WJ, Carr L and McAllister KW. Dynamic Stall Experiments on Oscillation Airfoils. AIAA Journal 1976; 14(1): 57-63.

21. McAllister KW, Carr L and McCrokskey WJ. Dynamic Stall Experiments on the NACA 0012 Airfoil. NASA Technical Paper (1100). 1978.

22. Akbari M and Price S. Simulation of dynamic stall for a NACA 0012 airfoil using a vortex method. Journal of Fluids and Structures 2003; (17): 855-874.

23. Wang S, Alam M, et al. Effects of Reynolds Number and Turbulent Intensity on a Low Reynolds Number Airfoil. Physics of Fluids 2014; 26(115107): 1-25.

24. Rinoie K, and Takemura N. Oscillating behaviour of laminar separation bubble formed on an aerofoil near stall. The Aeronautical Journal 2004; 108(1081): 153164.

25. Poirel D, Harris Y and Benaissa A. Self-sustained aeroelastic oscillations of a NACA 0012 airfoil at low-to-moderate Reynolds numbers. Journal of Fluids and Structures 2008; (24): 700-719.

26. Y. Z. Xu et al., Factors Influencing the Performance of Porous Wind Shields, Applied Mechanics and Materials, Vols. 321-324, pp. 799-803, 2013

27. Cummings RM, Morton SA, et al. Applied Computational Aerodynamics. 1st ed. 
New York: Cambridge University Press 2015.

28. Dowell EH, Clark R, Peters D, et. al. A Modern Course in Aeroelasticity. 4th ed. United States of America: Kluwer Academic Publishers, 2004.

29. Megson TH. Aircraft Structures for engineering students. 4th ed. Linacre House, Jordan Hill, Oxford: Elsevier, 2007.

30. Katz J. Race Car Aerodynamics - Designing For Speed 1st ed. Bently Publishers, 1995.

31. UIUC Applied Aerodynamics Group. UIUC Airfoil Data Site, http://mselig.ae.illinois.edu/ads/coord/n0012.dat (2018, accessed 3rd May 2019).

32. Anderson JD. Fundamentals of Aerodynamics. 5th ed. New York: Tata McGrawHill Education, 2010.

33. Jain S, Sitaram N, and Krishnaswamy S, Computational Investigations on the Effects of Gurney Flap on Airfoil Aerodynamics, International Scholarly Research Notices, vol. 2015, Article ID 402358, 2015. 